\title{
THE ESSCHER PREMIUM PRINCIPLE: A CRITICISM
}

\section{COMMENT}

\section{HANS U. GERBER}

ZEHNWIRTH (1981) contains some flaws. If

$$
H(X)=\frac{E\left[X e^{h x}\right]}{E\left[e^{h x}\right]} \quad(h>0)
$$

is the Esscher premium for a risk $X$, the loading is $H(X)-E(X)$ and not $h$ as Zehnwirth states. The first and third formulas on page 78 are wrong, since $o(h)$ is a quantity such that

$$
o(h) / h \rightarrow o \text { for } h \rightarrow 0 .
$$

A correct statement would have been that

$$
H(X)=E(X)+h / 2 \operatorname{Var}(X)+o(h)
$$

or simply that $H(X)$ is a continuous function of the parameter $h$. However, this continuity is not uniform in all risks, which is illustrated by (3). No matter how small $h$ is, there is always an $X$ such that the difference between $H(X)$ and $E(X)$ is substantial. In view of this what is the meaning of a statement like "... the Esscher premium is a small perturbation of the linearized credibility premium"?

Apart from some desirable properties (for example, additivity) the Esscher principle does indeed have some undesirable features. An example, communicated by De VyLDER, is the following: Let $0<z<1$, and let $X$ be a risk such that

$$
P(X=0)=P(X=z)=P(X=1)=1 / 3
$$

We consider the premium

$$
H(X)=\frac{z e^{h z}+e^{h}}{1+e^{h z}+e^{h}}
$$

a function of the parameter $z$, symbolically $f(z)=H(X)$. Obviously, it is 
highly desirable that $f(z)$ is an increasing function. However, this is not always the case: We find that

$$
f^{\prime}(0)=\frac{2-(h-1) e^{h}}{\left(2+e^{h}\right)^{2}}
$$

which is negative if $h$ is sufficiently large!

REFERENCE

Zehnwirth, B. (1981). The Esscher Premium Principle: A Criticism. Astin Bulletin, 12, 77-78. 